\title{
Determination of Wear, Friction Behavior and Characterization of Carbon Fiber Reinforced Epoxy Composites for Transport Applications
}

\author{
S.Sudhagar ${ }^{a^{*}}$, S.Sathees Kumar ${ }^{b}$ (1) \\ ${ }^{a}$ University College of Engineering Dindigul, Department of Mechanical Engineering, Tamil Nadu, \\ India. \\ ${ }^{b}$ CMR Institute of Technology, Department of Mechanical Engineering, Hyderabad, Telangana, India.
}

Received: June 21, 2020; Revised: October 26, 2020; Accepted: December 10, 2020

\begin{abstract}
Thermoplastics are finding the place in the current industrial sector due to its load bearing capacities. In this research, With the aid of pin on disc test set up, adhesive and abrasive wear behavior of leaf spring materials 30\% short carbon fiber reinforced epoxy (SF), 30\% long carbon fiber reinforced epoxy (LF) as well as Unreinforced epoxy (UF) are evaluated for automobile applications. Under multi pass abrasive wear condition, the effect of fiber reinforcement on plastic energy of deformation, matrix crystallinity and clogging behavior were investigated. The transient friction of Leaf spring materials was carried out under the load condition of $19.62 \mathrm{~N}$ and $28.43 \mathrm{~N}$. During the friction test, the effect of fiber length, fiber loading condition on the co-efficient of friction and its specific wear rate of the composite materials are also investigated. The increase in load during adhesive mode for all the materials, specific wear rate and wear volume of the test materials also increased. Furthermore, the fiber- matrix interface, fractured surfaces were observed thoroughly through Scanning Electron microscopic (SEM) morphology.
\end{abstract}

Keywords: Carbon fiber, Short carbon fiber, Long carbon fiber, Transient friction, Wear, SEM.

\section{Introduction}

In the current scenario, thermoplastic started replacing thermoset plastic for its ecological nature and high mass production capability. discontinuous long fiber reinforced thermoplastics (LFRT) have shown significant application in replacing metals, short fiber reinforced thermoplastics, thermoset sheet molding and bulk molding composites. A leaf spring is the main component commonly used for suspension in every wheeled vehicle. Leaf springs were used in automobiles to isolate shocks and vibrations transmitted due to the uneven road conditions. Due to the superior performance of specific strain energy, damping and corrosion resistance of composite materials over steel, Composite leaf springs were being broadly used in light weight vehicles, passenger cars, heavy tank trailer suspension systems and in vibrating machineries ${ }^{1,2}$. Composite leaf springs are being broadly used in light weight vehicles, passenger cars, heavy tank trailer suspension systems and in vibrating machineries ${ }^{3-5}$ because of the superior performance of specific strain energy, damping and corrosion resistance of composite materials over steel. The following techniques are followed for manufacturing composite leaf springs; filament winding, compression moulding, pultrusion and hand lay-up vacuum bag process. Due to utilization of continuous fibers, excellent mechanical properties are provided by filament winding process. Nevertheless, the higher degree of design flexibility is constrained by the leaf springs with varying thickness and changing contours. Various shapes and sizes of leaf springs

*e-mail: ssudhagar6@gmail.com can be produced by compression moulding; however, the mass production capability ${ }^{6}$ is limited by manufacturing time involved in compression moulding. The mass production features of composite leaf springs ${ }^{7}$ are limited by hand lay-up vacuum bag process involves high level of skills for mixing and control of resin contents. Once, the fabrication of composite leaf spring manufactured by using epoxy, polyester and polyimide resins. For the reinforcement of leaf springs, unidirectional E-glass, S-glass and carbon fibers were used. The improved potential for commercial leaf spring applications over S-glass and carbon fibers is provided by the finest blend of cost and performance of E-glass fibers. In the matrix resins, epoxy resins based on cost and ease of processability $^{8}$ was dominated by polyester. Lhymn et al. ${ }^{9}$ investigated the abrasive wear behavior of PEEK/polypropylene sulfide (PPS) blended thermoplastic polymer reinforced with short carbon and glass fiber, and stated that the wear rate was sensitive to the orientation of the fiber axis with regards to the sliding direction. Cirino et al. ${ }^{10}$ examined the abrasive wear behavior of unidirectional continuous glass fiber and focused the effect of operating variables on the polymer matrices. McGee et al. ${ }^{11}$ examined the importance of fiber matrix adhesion in reducing the abrasive wear resistance in the graphite filled nylon matrix configuration. Bijwe et al. ${ }^{12}$ stated that the abrasive wear characteristics are enhanced notably by the addition of glass, aramid and carbon fibers in polyetherimide composites and reported the influence of operating parameters on abrasive wear resistance. Barkoula and Karger-Kocsis ${ }^{13}$ stated that the fiber length did not affect 
the erosive wear at high impact angle, through his investigation over the erosive wear of polypropylene reinforced with glass fibers with length of 2 and $10 \mathrm{~mm}$ and Tong et al. ${ }^{14}$ reported that the abrasive wear resistance was improved by hindering the easy deduction of fibers from high molecular weight polyethylene (HMWPE) matrix through the influence of aspect ratio on wear resistance; with the addition of wollastonite fibers with a large aspect ratio. Suresha et al. ${ }^{15}$ examined the worn out surface of carbon epoxy and glass epoxy composites by means of scanning electron microscope and envisaged the rupture of more glass fibers compared to carbon fiber because of improved interfacial adhesion between epoxy and carbon fiber. Kanagaraj et al. ${ }^{16}$ identified that the decrease in wear coefficient and wear volume when the carbon nano tubes are added in ultrahigh molecular weight polyethylene (UHMWPE) polymer and stated that wear volume increases with regards to the sliding distance. Reason for using carbon fibers in this work, carbon fibers have great specific strength, high modulus, good in fatigue resistance and dimensional stability and lower density fibers composite materials have their high strength and stiffness. If it combined with low density, when compared with bulk materials, allows for a weight reduction in the finished part.

In this research work, the wear resistance under adhesive condition is essential for leaf spring material, as the leaf spring materials such as SF, LF and UF has subjected to contact loads. The transient friction of leaf spring materials has carried out under the load condition of 19.62 N. During the friction test, the effect of fiber length, fiber loading condition on the co-efficient of friction and its specific wear rate of the composite materials are performed. However, with the assistance of Scanning Electron microscopic morphology, the adhesive wear mechanisms of selected thermoplastic composites were identified.

\section{Experimental work}

\subsection{Fabrication Process of the composites}

For the development of thermoplastic leaf springs, unreinforced epoxy (UF), 30\% short carbon fiber reinforced epoxy (SF) and 30\% long carbon fiber reinforced epoxy (LF) were taken into account. Leaf spring material characteristics like friction and damping were also assessed before the development of leaf spring, for the above-mentioned materials. From extrusion process, SF pellets are prepared where fibers are arbitrarily oriented. LF pellets were prepared from pultrusion technique where fibers are well oriented along with the pellet length. Pellets were obtained from Saint-Gobain India, according to the product data sheet, same molecular weight was being possessed by the base resin epoxy and coupling agent having same amount of silane type was used for the carbon fibers in LF and SF pellets. Void is assumed to be same for both short and long fiber reinforced composites since the amount of void content is significantly small and intricate to quantify by the available test facility. Material behaviour deliberations were partial only to the fiber length since the examined reinforced materials have the same type and amount of coupling agent. To remove excess moisture, initially pellets were maintained at $80^{\circ} \mathrm{C}$ for $2 \mathrm{~h}$, and later injection moulded in to cylindrical specimens as per ASTM
D 6110 standard. To investigate the adhesive friction wear performance for the chosen leaf spring materials, pin on disc tribometer exposed and utilized and as per ASTM G 99 standard, the testing was also completed. According to cylindrical pin of $5 \mathrm{~mm}$ diameter and $20 \mathrm{~mm}$ length, specimens relating to ASTM D 6110 standard were machined ${ }^{17-19}$. Physical properties of carbon fiber as shown in Table 1.

\subsection{Adhesive friction wear}

The adhesive friction wear performance for the chosen leaf spring materials, pin on disc tribometer exposed and utilized and as per ASTM G 99 standard, the testing was also carried out. According to the ASTM D 6110 standard the cylindrical pin of $5 \mathrm{~mm}$ diameter and $20 \mathrm{~mm}$ length specimens are machined.

In support of the counter material, stainless steel disc (AISI 314) having centre line average surface roughness ( $\mathrm{Ra}$ ) of $0.1433 \mu \mathrm{m}$ was utilized. As per ASTM D 2240 standard, initial hardness of the test, materials were calculated. By means of the force transducer fixed on the loading lever arm, the friction force was calculated and by using a computerbased data acquisition system, it was stored. At 19.62 and $28.43 \mathrm{~N}$ normal loads with a stable sliding velocity of $0.5 \mathrm{~m} / \mathrm{s}$, and sliding distance of $3000 \mathrm{~m}$ carry out adhesive friction and wear performances. In the dry conditions $\left(32 \pm 3^{\circ} \mathrm{C}, \mathrm{RH}\right.$ $57 \pm 6 \%$ ) tests were executed. On behalf of friction force and displacement measurement, data sampling rate was set aside as $1 \mathrm{~Hz}$. Toward polish the test specimen to the standard surface roughness value of $0.9 \mathrm{Ra}$, silica carbide emery paper was used well. By applying an electronic balance of $0.1 \mathrm{mg}$ accuracy, test specimen mass was calculated. At similar test circumstances, as a minimum of three tests were conducted and the average values of frictional force and mass loss were used for the supplementary investigation. By using Equation 1, the specific wear rate $(\mathrm{K})$ was calculated,

$$
K=\frac{\left(m_{1}-m_{2}\right)}{P S \rho_{g}} \times 1000
$$

Here $m_{1}$ and $m_{2}$ are specimen mass before and after testing in $\mathrm{g}, \rho_{\mathrm{g}}$ is the density of the specimen $\left(\mathrm{g} / \mathrm{cm}^{3}\right), \mathrm{P}$ is the normal load in $\mathrm{N}$ and $\mathrm{S}$ is the sliding distance in $\mathrm{m}$. Subsequent to sputtering with gold coating, worn out surfaces of the pins were identified with $\mathrm{SEM}^{20-22}$.

\subsubsection{Mechanism on the Transient Friction}

It was made clear that there is an increase in friction coefficient in the initial period $(1500 \mathrm{~m})$ from the Figure 1, after that a steady state was obtained by the friction coefficient. Commonly, in influencing wear mechanism, a significant role is played by the formation of transfer film on the counter material. A steady state was reached by coefficient of friction on the counter face material only after the formation of trans fiber film Figure 2a-c.

Due to the asperities deformation, the actual area of contact increases as close as to the visible area of contact when the sliding distance increases. Primarily, their opposing asperities with maximum height come in contact when two surfaces approach each other. New pairs of asperities with 
lesser height create contact forming individual spots when the time increases.

This behavior was established by the measurement of surface roughness before and after sliding was indicated in the Figure 3. When compared to UFPE, the reduction of surface roughness in fiber reinforced epoxy was impeded by the existence of fibers and the breakage of fibers during sliding over the steel counter face therefore when compared to its counterparts, Ra for soft UFPE was least. For the principal abrasive wear, more number of asperities contributes in

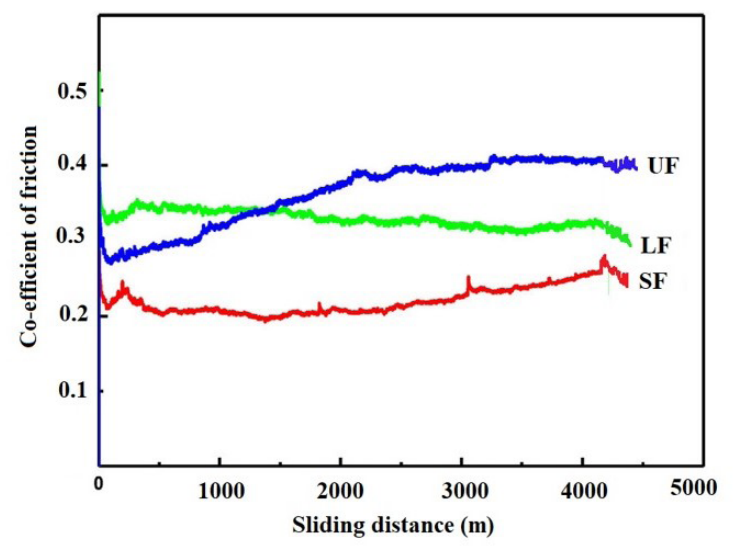

Figure 1. Coefficient of friction at $19.62 \mathrm{~N}$ for materials under various loading parameters. the early period of contact. The number of asperities was reduced by the increase in sliding distance/time, in which adhesion wear leads. Thus, for all the test materials, initially there was an increase in coefficient of friction owing to the dominant abrasive friction and later reaches constant value owing to the principal adhesive friction ${ }^{23,24}$.

\subsection{Effect of Fiber Length on the Friction}

A considerable role is played by the fiber length in influencing the frictional coefficient of the examined materials after running in period $(1500 \mathrm{~m})$. Owing to the adhesion between the contacting surfaces, the material transfer phenomenon is become principal. By mechanical interlocking of polymer material to the metal asperities, the transfer of material is directed well. In general, from weaker to stronger material the particle shift is occurred. In the case of carbon fiber reinforced epoxy than unreinforced epoxy, transfer film thickness was discovered to be thin LF showed thin transfer film than SF among the reinforcements, owing to its superior fiber matrix bonding. Similar behavior of transfer film for the graphite filled composites, in which the frictional coefficient increased initially after that dropped to constant value, therefore the formation of a graphite transfer film on the counter face, was confirmed by it. LF exhibited greater mechanical characteristics than that of LF compared to SF despite identical category of fibers and identical quantity of coupling agent. In common, the interfacial shear strength is directly proportional to fiber length. The

Table 1. Physical properties of carbon fiber.

\begin{tabular}{cccccc}
\hline $\begin{array}{c}\text { Tensile strength } \\
(\mathbf{M P a})\end{array}$ & $\begin{array}{c}\text { Tensile modulus } \\
\mathbf{( G P a )}\end{array}$ & $\begin{array}{c}\text { Elongation } \\
\mathbf{( \% )}\end{array}$ & $\begin{array}{c}\text { Density } \\
\left(\mathbf{g} / \mathbf{c m}^{\mathbf{3}}\right)\end{array}$ & $\begin{array}{c}\text { Compressive } \\
\text { strength } \mathbf{( G P a )}\end{array}$ & $\begin{array}{c}\text { Shear modulus } \\
(\mathbf{G P a})\end{array}$ \\
\hline 4890 & 390 & 1.9 & 1.79 & 1.25 & 15.8 \\
\hline
\end{tabular}
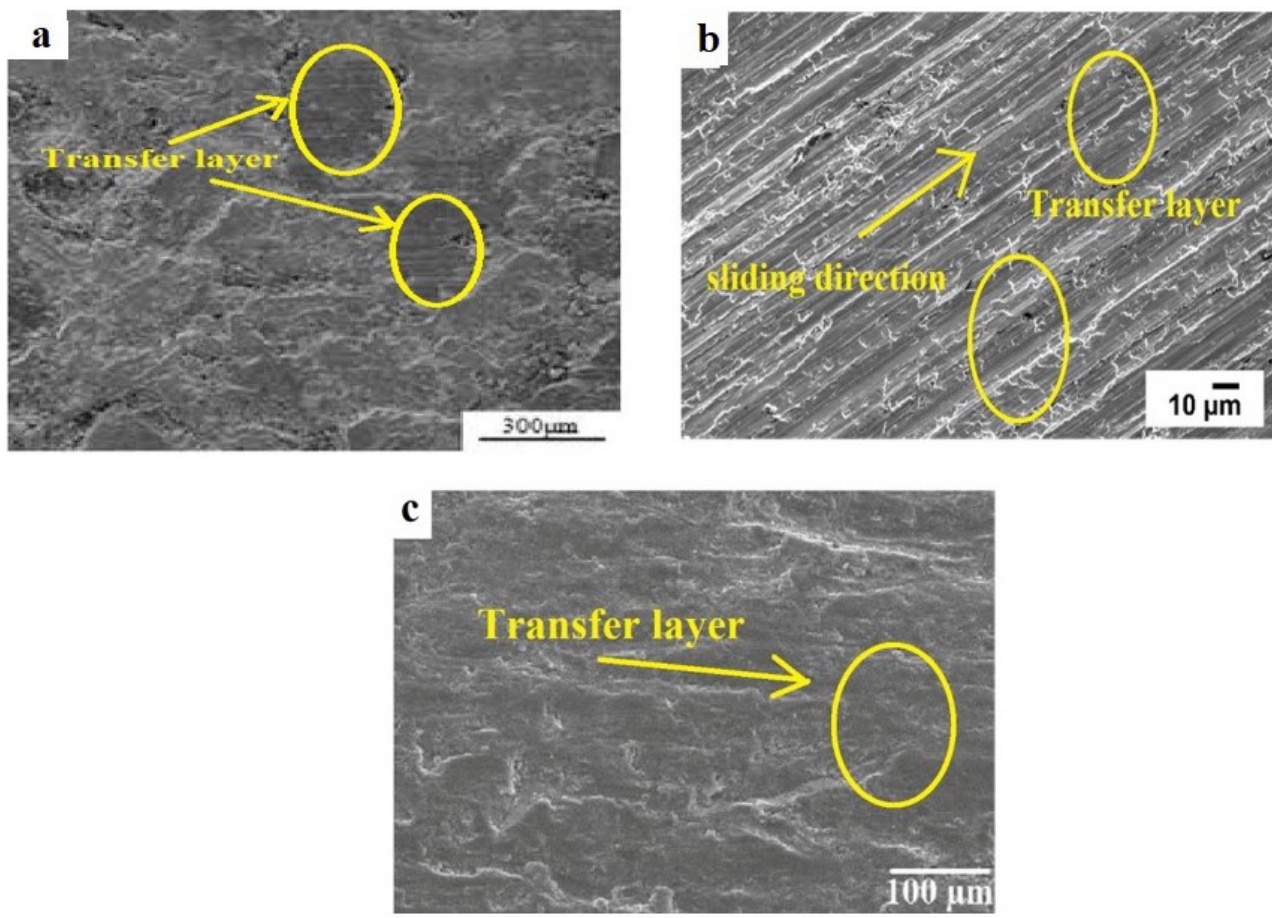

Figure 2. Transfer layers formed on stainless steel at $29.43 \mathrm{~N}$ for the material (a) UFPE, (b) SF (c) LF. 
fiber should have a minimum length for effective composite strengthening, through which an efficient load transfer is feasible. Fibers which are shorter than this decisive length do not serve as load-bearing component. The fiber-matrix bonding was proved to be better which ultimately enhanced its mechanical properties since the fiber length in LF is three times more than SF. The above deliberations were authenticated by carrying out tensile test on fiber matrix bonding, on the selected material and to comprehend the fiber- matrix interface, fractured surfaces were examined thoroughly. At atmospheric condition $\left(23{ }^{\circ} \mathrm{C}\right.$ and $\left.50 \% \mathrm{RH}\right)$ the tests were executed. When the gauge length of the test material was $50 \mathrm{~mm}$, the crosshead was moved at $1 \mathrm{~mm} / \mathrm{min}$ speed. The stress-strain curve of the test materials is shown

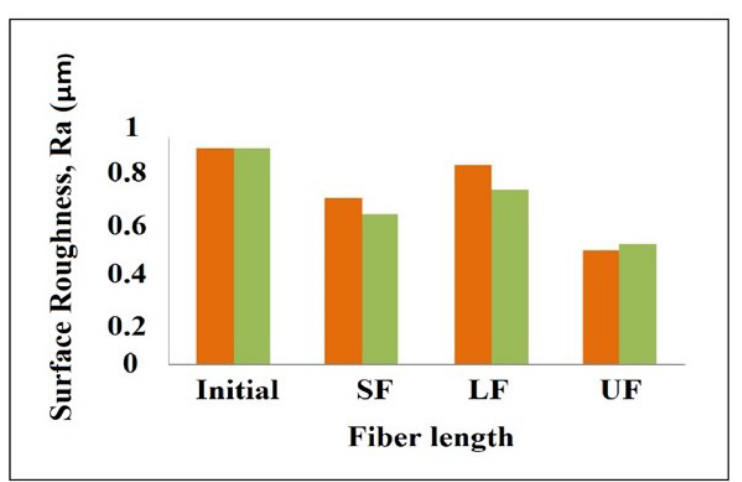

Figure 3. Influence of the fiber length on surface roughness.

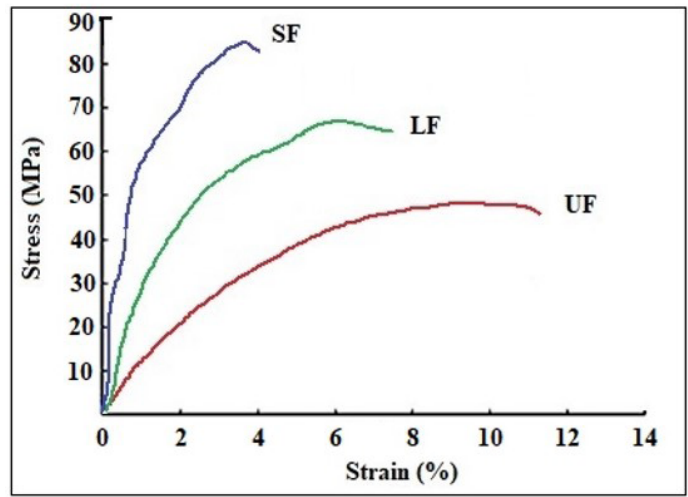

Figure 4. Stress-strain curve of pure and reinforced epoxy composites.

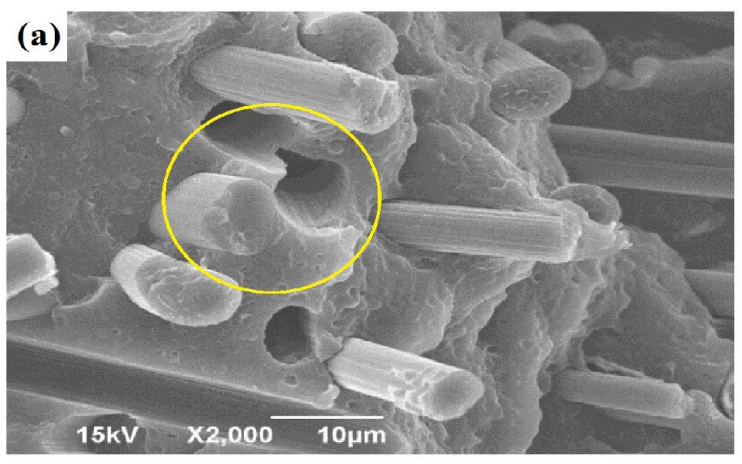

in Figure 4. The addition of reinforcement increased the material modulus and strength. SEM of LF showed hardly fiber pull out whereas SEM of SF exhibited more fiber pull out failures as indicated in Figure 5 and Figure 6. Hence, surface area of the fiber is increased by the increase in fiber length of LF material and fine interfacial bonding strength is also provided with the matrix and thus, fiber pullout was completely avoided.

When compared to short fibers in SF, the steady-state frictional coefficient significantly is lowered by the presence of long fibers in the LF. The test material hardness and fiber reinforcement increased material hardness are shown in the Figure 7.

\subsection{Effect on the fiber loading condition on the coefficient of friction and its wear rate}

In the Figure 8, the influence of load on the coefficient of friction has revealed clearly. All the considered test materials, the friction coefficient decreased slightly with increase in applied load. Owing to the low sliding resistance offered by the specimen asperities at higher loading condition was the sole reason for this behavior. Specific wear rate of all the test materials increased with the increase in PV despite the decrease in friction coefficient is shown in Figure 9. Due to the higher normal load acting on the specimen there was an increase in explicit wear rate. Less specific wear rate than unreinforced epoxy has revealed in both the load conditions/PV values, and reinforced epoxy.

The addition of carbon fiber to epoxy abridged the wear rate. LF exhibited less specific wear than SF and UF among the reinforced epoxy. The enhanced wear resistance in long carbon fiber epoxy was notified and owing to more surface area of the fiber in contact, the importance of higher aspect ratio in developing the adhesion between fiber and the matrix. The enhanced wear resistance in carbon fiber reinforced epoxy with the addition of maleic anhydride was envisaged as well as the enhanced fiber matrix bonding strength between fiber and matrix. Fine worn particles of carbon fiber was embedded with the smooth shiny surface, upon which the sliding smooth surface was created and that significantly abridged the specific wear rate than other two considered materials were all revealed in the Figure 10a.

The capability of long reinforced carbon fibers in enduring the tough protuberance owing to counter face is clearly

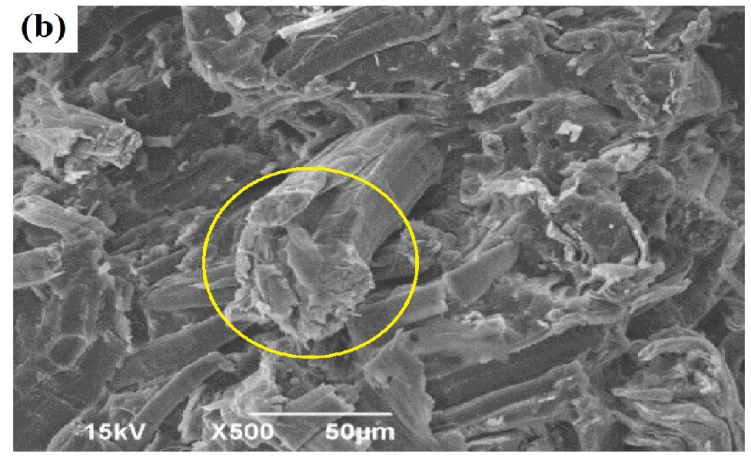

Figure 5. SEM of fractured surface of SF composites. 


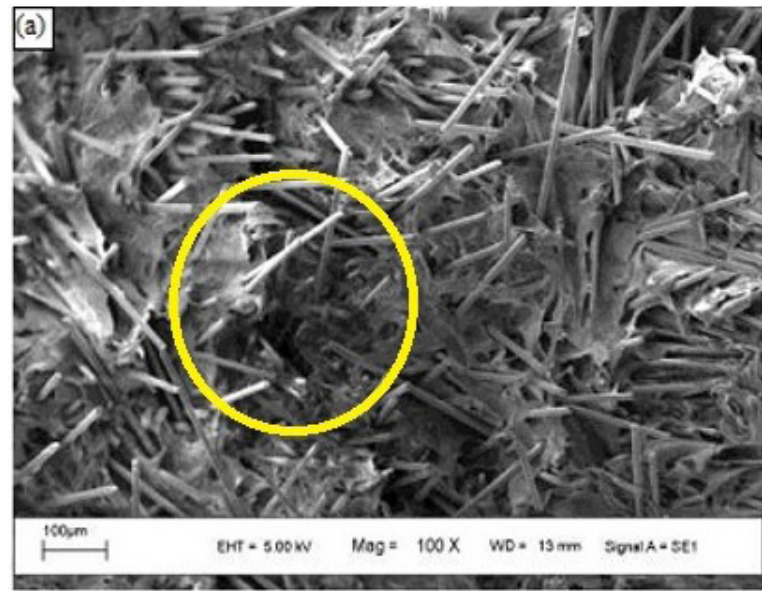

Figure 6. SEM of fractured surface of LF composites.

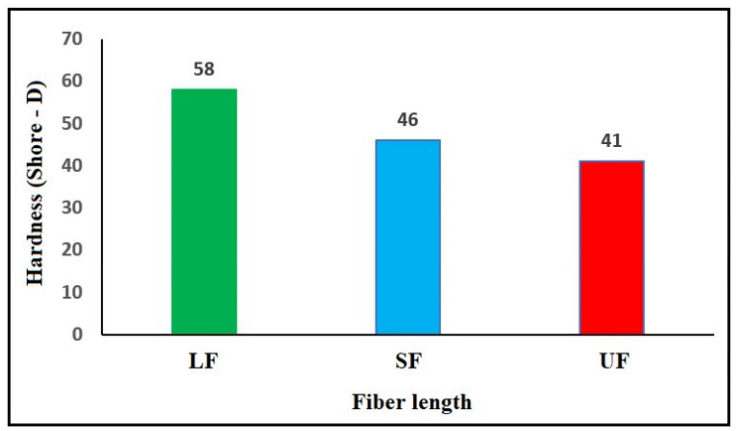

Figure 7. Hardness of the composites.

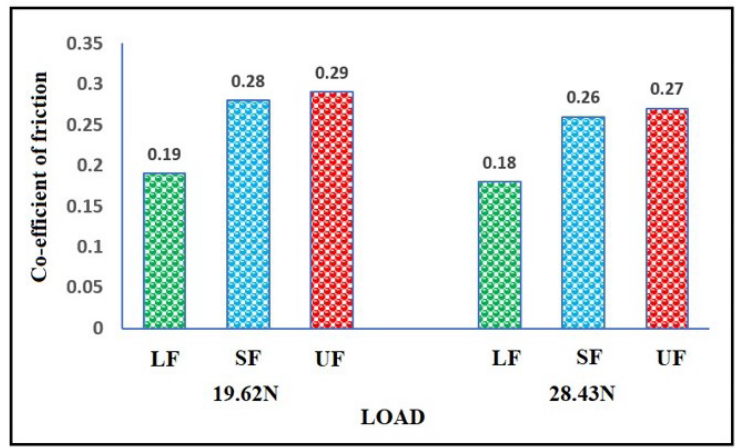

Figure 8. Influence of the load Vs coefficient of friction of the different composite materials.

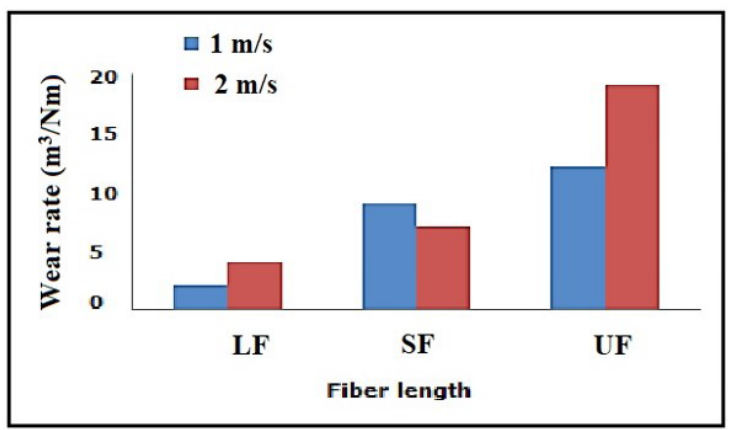

Figure 9. Influence of velocity Vs specific wear rate of the composite materials.

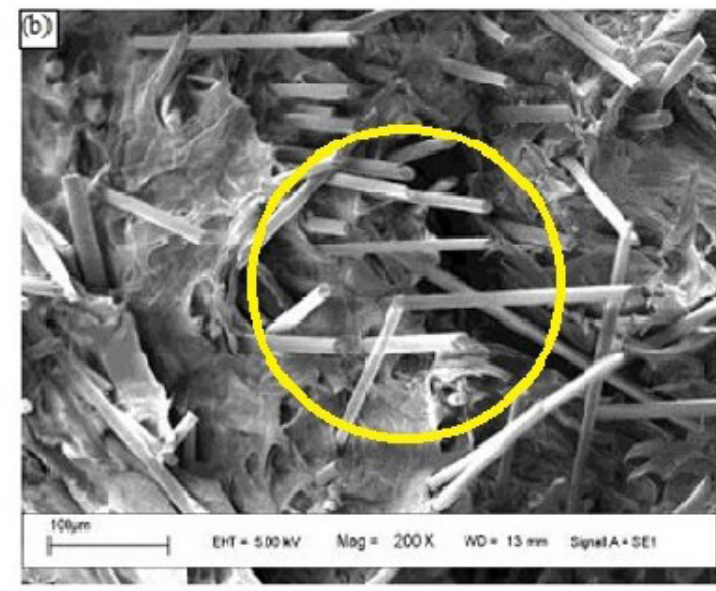

represented by the scratch marks in the sliding direction of long fiber reinforced epoxy as shown in Figure 10b.

\subsection{Wear Mechanisms of the composite specimens}

Figure 11a owing to de-bonding in the surface of SF, the fiber pullout was visibly indicated, the existence of more fiber ends as well as poor fiber matrix bonding strength were the reasons for this behavior. As a third body abrasive, the accumulated debris at the SF specimens was acted and the specific wear rate of SF as LF was also increased as represented in the Figure 11b. The frail bond between fiber and matrix was confirmed by the fibrils formed at SF as shown in the Figure 11c. Poor wear resistance was the outcome of the behavior of abrasive reinforced carbon fibers which were freely bounded to PTFE matrix. The effectiveness with which the fiber reinforces the matrix increases when the fiber length increases as well.

Fiber breakage failure can be anticipate while the load surpasses the greatest permissible fiber strength. As a stress concentration region, which assisted fiber breakage, the configuration of grooves and some patches of tranfiberer film on the LF surface were served. Due to the adequately larger fiber length and enhanced fiber matrix bond, LF displayed broken fibers as indicated in the Figure 12a and fiber entanglement as represented in the Figure 12b in the current case. The good wear resistance is this sort of fiber breakage/entanglement in a surface leaves the result of the surface comparatively uninterrupted. The ductile wear was exhibited by unreinforced epoxy as shown in Figure 13a-b moreover it was subjugated by micro ploughing and as a result of the asperities of the mating steel disc, deep grooves were caused.

On the wear performance of leaf spring materials, adhesive wear performance of leaf spring materials confirmed the function of fiber length. Compared to that of short fiber reinforced and unreinforced material, the enhanced fiber matrix bonding, hardness and presence of less fiber ends in long fiber reinforced material reduced specific wear rate significantly. In adhesive mode, UF exhibited micro ploughing. In SF material at adhesive and abrasive mode fiber pullout and micro cutting were observed respectively. Under adhesive mode, LF exhibited fiber breakage. 

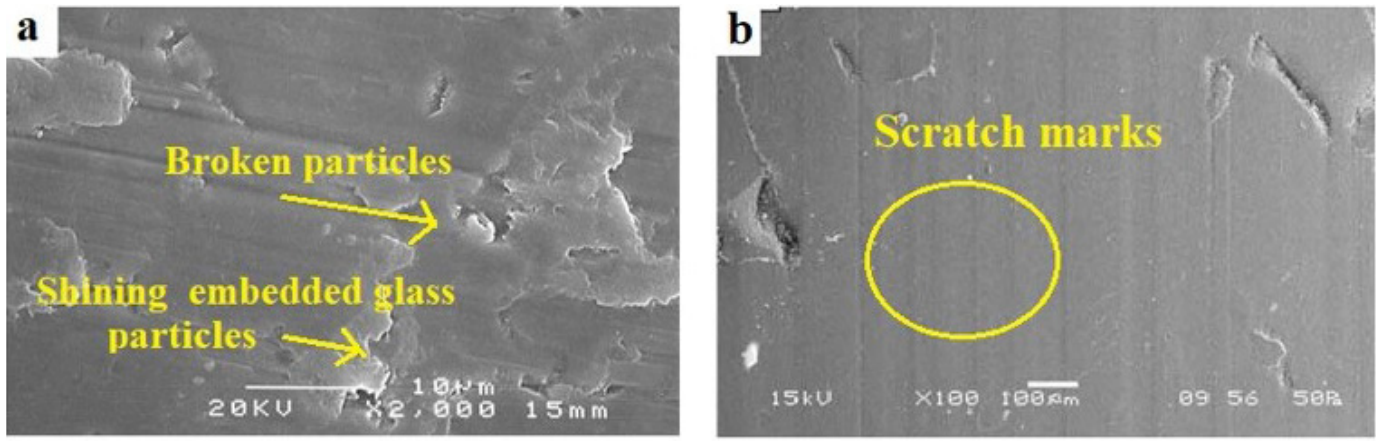

Figure 10. Morphological Structure of long fiber reinforced epoxy at 29.43N.
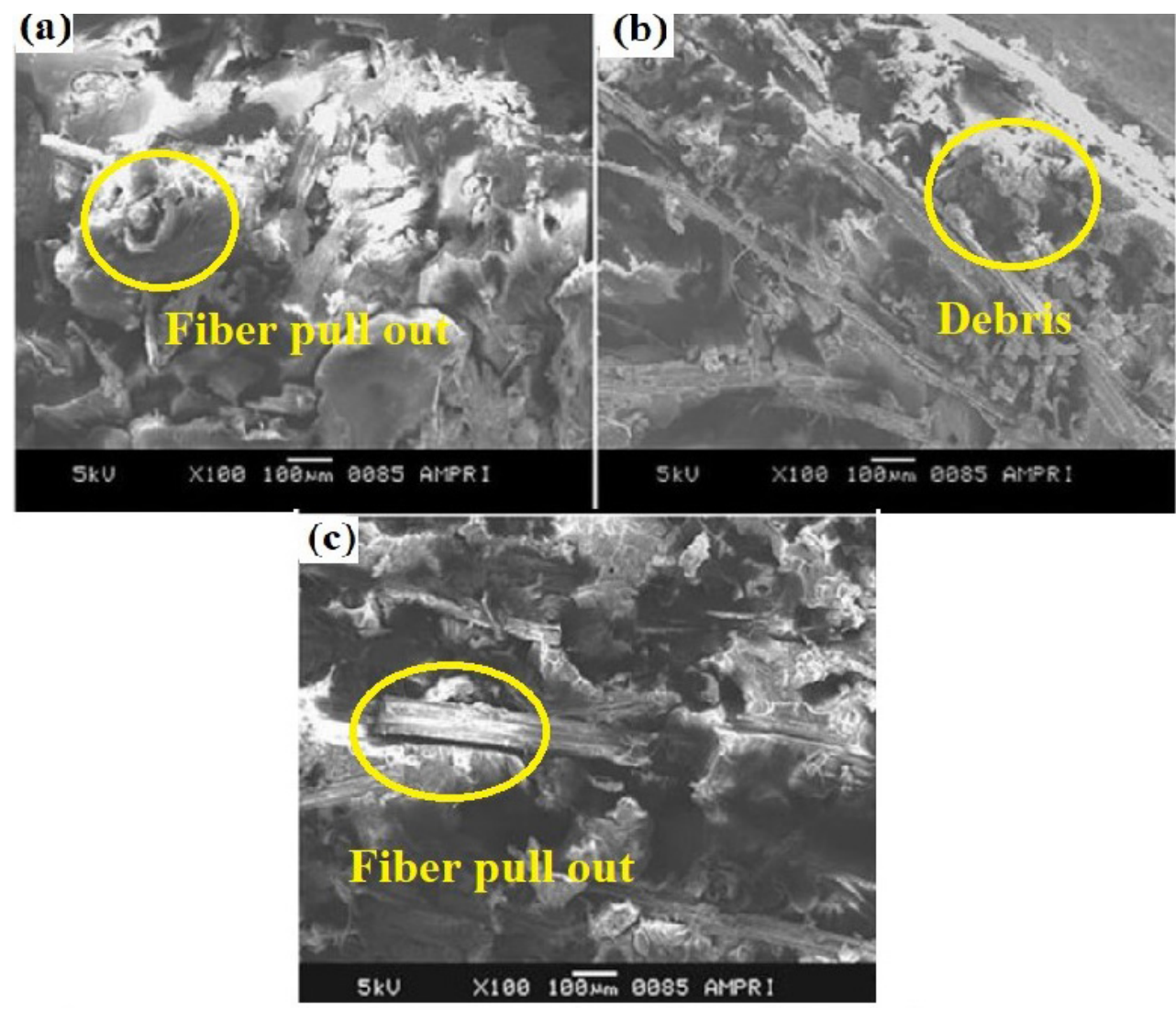

Figure 11. (a-c) SEM image of short fiber reinforced epoxy at 29.43N.
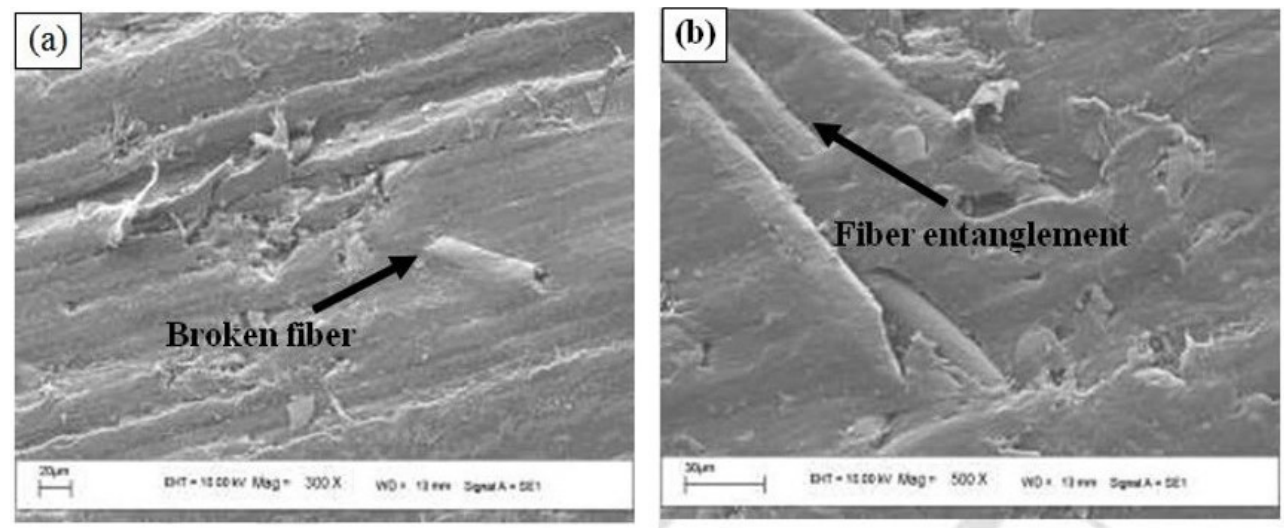

Figure 12. (a-b) Morphological Structure of long fiber reinforced epoxy at $29.43 \mathrm{~N}$. 

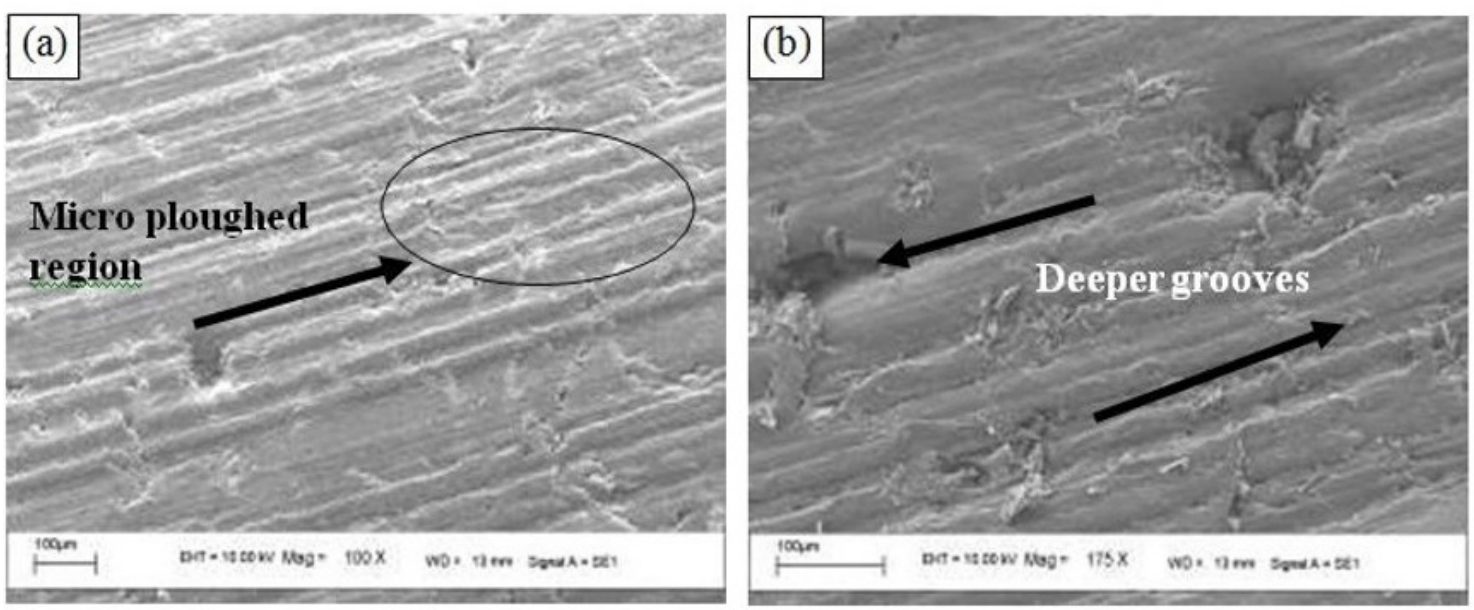

Figure 13. (a-b) SEM image of unreinforced epoxy material at $29.43 \mathrm{~N}$.

\section{Conclusion}

For the leaf spring materials under adhesive mode using pin on disc test configuration, friction and wear characteristics were studied. The wear performance was interrelated with plastic deformation energy and crystallite size of the test materials.

- The hardness improved by fiber reinforcement and the coefficient of friction than unreinforced material reduced effectively. In adhesive mode when compared to SF, less specific wear rate shown by reinforcement LF.

- When compared to that of SF and UF, improved fiber matrix bonding and presence of less fiber ends in LF improved wear resistance. The increase in load during adhesive for all the materials, specific wear rate and wear volume of the test materials also increased.

- $\quad$ Significantly, friction-wear behavior was affected by the transfer film formation and clogging on the counter face during adhesive mode. In unreinforced material, transfer layer and clogged layer were found to be more distinct than reinforced material.

- By the resulted superior wear resistance compared to that of SF and UF, LF exhibited was improved.

- Due to the dominant failure morphology, UF, SF and LF exhibited micro ploughing, fiber pullout and fiber breakage respectively under abrasive mode.

- This type of composites can be used for automotive applications.

\section{References}

1. Beardmore P. Composite structures for automobiles. Compos Struct. 1986;5(3):163-76. http://dx.doi.org/10.1016/02638223(86)90001-2.

2. Morris CJ. Composite integrated rear suspension. Compos Struct. 1986;5(3):233-42. http://dx.doi.org/10.1016/02638223(86)90005-X.

3. Sancaktar E, Gratton M. Design, analysis, and optimization of composite leaf springs for light vehicle applications. Compos Struct. 1999;44(2-3):195-204. http://dx.doi.org/10.1016/S02638223(98)00136-6.
4. Rajendran I, Vijayarangan S. Optimal design of a composite leaf spring using genetic algorithms. International Journal of Computers and Structures. 2001;79(11):1121-9. http://dx.doi. org/10.1016/S0045-7949(00)00174-7.

5. Hou JP, Cherruault JY, Jeronimidis G, Mayer R. Design, testing, and simulation of fibre composite leaf springs for heavy axle loads. J Strain Anal Eng Des. 2005;40(6):497-504. http://dx.doi. org/10.1243/030932405X30704.

6. Mallick P. Composites engineering handbook. Boca Raton: CRC Press; 1997. http://dx.doi.org/10.1201/9781482277739.

7. Crawford RJ. Plastics engineering. Oxford: Butterworth Heinemann; 1998.

8. Beardmore P, Johnson CF. The potential for composites in structural automotive applications. Compos Sci Technol. 1986;26(4):25181. http://dx.doi.org/10.1016/0266-3538(86)90002-3.

9. Lhymn C, Tempelmeyer KE, Davis PK. The abrasive wear of short fibre composites. Composites. 1985;16(2):127-36. http:/ dx.doi.org/10.1016/0010-4361(85)90619-6.

10. Cirino M, Pipes B, Friedrich K. The abrasive wear behaviour of continuous fibre polymer composites. J Mater Sci. 1987;22(7):2481-92. http://dx.doi.org/10.1007/BF01082134.

11. McGee AC, Dharan CKH, Finnie I. Abrasive wear of graphite fiber-reinforced polymer composite materials. Wear. 1997;114(1):97107. http://dx.doi.org/10.1016/0043-1648(87)90019-6.

12. Bijwe J, Indumathi J, John Rajesh J, Fahim M. Friction and wear behavior of polyetherimide composites in various wear modes. Wear. 2001;249(8):715-26. http://dx.doi.org/10.1016/ S0043-1648(01)00696-2.

13. Barkoula NM, Karger-Kocsis J. Effects of fibre content and relative fibre-orientation on the solid particle erosion of GF/PP composites. Wear. 2002;252(1-2):80-7. http://dx.doi.org/10.1016/ S0043-1648(01)00855-9.

14. Tong J, Ma J, Jiang M. Effects of the wollastonite fiber modification on the sliding wear behavior of the UHMWPE composites. Wear. 2003;255(1-6):734-41. http://dx.doi. org/10.1016/S0043-1648(03)00221-7.

15. Suresha B, Chandramohan G, Siddaramaiah, Samapthkumaran P, Seetharamu S. Three-body abrasive wear behaviour of carbon and glass fiber reinforced epoxy composites. Mater Sci Eng A. 2007;443(1-2):285-91. http://dx.doi.org/10.1016/j. msea.2006.09.016.

16. Kanagaraj S, Mathew MT, Fonseca A, Oliveira MSA, Simoes JAO, Rocha LA. Tribological characterisation of carbon nanotubes/ultrahigh molecular weight polyethylene composites: the effect of sliding distance. Int J Surface Sci Eng. 2010;4(46):305. http://dx.doi.org/10.1504/IJSURFSE.2010.035138. 
17. Sathees Kumar S, Kanagaraj G. Investigation of characterization and mechanical performances of $\mathrm{Al} 2 \mathrm{O} 3$ and $\mathrm{SiC}$ reinforced PA6 hybrid composites. J Inorg Organomet Polym Mater. 2016;26(4):788-98. http://dx.doi.org/10.1007/s10904-016-0387-6.

18. Kumar SS, Kanagaraj G. Evaluation of mechanical properties and characterization of silicon carbide-reinforced polyamide 6 polymer composites and their engineering applications. Int J Polym Anal Charact. 2016;21(5):378-86. http://dx.doi.org/1 0.1080/1023666X.2016.1160671.

19. Sathees Kumar S, Kanagaraj G. Investigation on mechanical and tribological behaviors of PA6 and graphite-reinforced PA6 polymer composites. Arab J Sci Eng. 2016;41(11):4347-57. http://dx.doi.org/10.1007/s13369-016-2126-2.

20. Sathees Kumar S, Kanagaraj G. Experimental investigation on tribological behaviours of PA6, PA6-reinforced A12 O 3 and PA6-reinforced graphite polymer composites. Bull Mater Sci. 2016;39(6):1467-81. http://dx.doi.org/10.1007/s12034-016-1296-6.
21. Sathees Kumar S, Kanagaraj G. Effect of graphite and silicon carbide fillers on mechanical properties of PA6 polymer composites. Journal of Polymer Engineering. 2017;37(6):54757. http://dx.doi.org/10.1515/polyeng-2015-0441.

22. Sudhagar S, Raja VM, Sathees Kumar S, Samuel AJ. The wear behavior and service life of Madar and Bauhinia Racemosa reinforced polyester hybrid composites for gear applications. Mater. Today Proceedings. 2019;19(Pt 2):589-93.

23. Raja VM, Kumar SS. Determination of static and fatigue characteristics of carbon fiber reinforced polyester composites for automobile applications. Mater Res. 2019;22(6):e20190458. http://dx.doi.org/10.1590/1980-5373-mr-2019-0458.

24. Sathees Kumar S. Effect of natural fiber loading on mechanical properties and thermal characteristics of hybrid polyester composites for industrial and construction fields. Fibers Polym. 2020;21(7):1508-14. http://dx.doi.org/10.1007/s12221-0209853-4. 